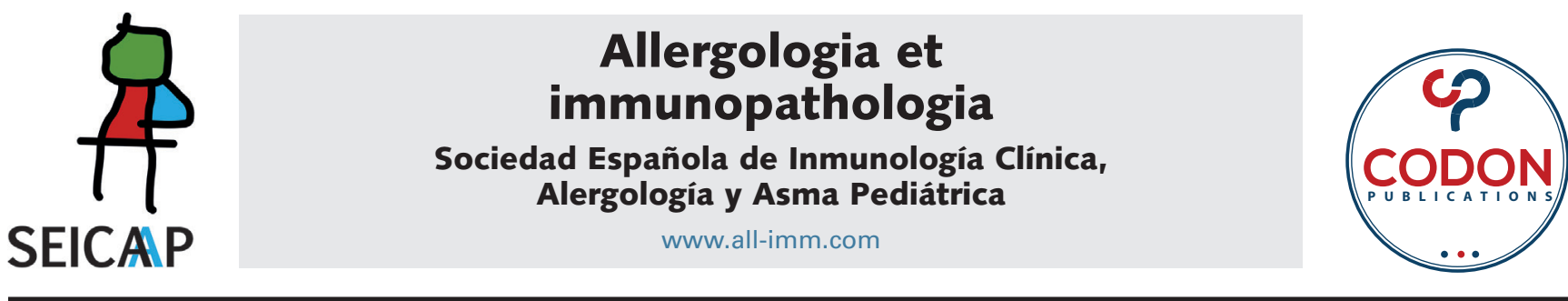

\title{
Familial Mediterranean fever in the pediatric population
}

\author{
Pilar Llobet-Agullóa,b*, Laura Sanromà-Nogués ${ }^{b}$, Isabel Maria Salguero-Pérez ${ }^{b}$, \\ Juan I. Arósteguic,de, Sonia Corral-Arboledas ${ }^{b}$, Maria Teresa Coll-Sibina ${ }^{b}$, \\ Lluìsa Masiques-Mas ${ }^{\mathrm{b}}$
}

\author{
aSpanish Society of Clinical Immunology, Allergology and Pediatric Asthma, Spain \\ ${ }^{b}$ Department of Pediatrics, Hospital General de Granollers, Barcelona, Spain \\ 'Department of Immunology, Hospital Clínic de Barcelona, Barcelona, Spain \\ 'Institut d'Investigacions Biomèdiques August Pi I Sunyer, Barcelona, Spain \\ eUniversitat de Barcelona, Barcelona, Spain
}

Received 14 May 2021; Accepted 26 August 2022

Available online 1 January 2022

\section{KEYWORDS \\ Amyloid A; \\ Anti-IL1; \\ Colchicine; \\ Familial \\ Mediterranean fever; \\ Fever}

\begin{abstract}
Familial Mediterranean fever (FMF) is the most frequent autoinflammatory disorder characterized by short, repeated, and self-limiting crises of fever and serositis. The disease was described as autosomal recessive hereditary transmission secondary to variants of the MEFV (MEditerranean FeVer) gene, even though a variable proportion of patients only present a heterozygous variant. FMF is very common in certain ethnic groups (Turkish, Armenian, Arab, and Jewish), even though it has been described throughout the Mediterranean and elsewhere in the world. The clinical manifestations are variable, with secondary amyloidosis being the most serious complication of the disorder. Treatment and prophylaxis are mainly based on the administration of colchicine, which prevents the crises and avoids complications in most cases. This study reviews the course of seven pediatric patients diagnosed with FMF during the period 2010-2018 at a district hospital. Most of the patients were of Caucasian origin, with onset at an early age in the form of fever as the main symptom, and some patients moreover presented less frequent manifestations (pericardial effusion, sensorineural hearing loss). Two cases presented plasmatic amyloid A protein elevation that subsided with the treatment. All the patients initially received colchicine, and one of them required prescription of anakinra, which was replaced by canakinumab due to a serious adverse reaction. There were no cases of consanguinity, and all the patients were of Mediterranean origin. The subjects showed a favorable course over the years, which was attributed to the early diagnosis and treatment provided.
\end{abstract}

(c) 2022 Codon Publications. Published by Codon Publications.

*Corresponding author: Pilar Llobet Agulló, Spanish Society of Clinical Immunology, Allergology and Pediatric Asthma. Department of Pediatrics, Hospital General de Granollers, Barcelona, Spain. Email address: pllobet@fphag.org 


\section{Introduction}

Familial Mediterranean fever (FMF) is a genetic disorder characterized by short, repeated, and self-limiting crises of fever and serositis. ${ }^{1,2}$ It is the most common hereditary autoinflammatory disease. ${ }^{3-5}$ FMF can simulate many other disease conditions, and its most serious complication is the appearance of secondary amyloidosis, which mainly affects the kidneys, causing nephrotic syndrome and renal failurethough other organs can also be affected. ${ }^{6}$

While the highest prevalence of FMF is found in Arab, Turkish, Armenian, and Sephardic Jewish populations, and the disease is being frequent in parts of Italy, Greece, and Spain, all ethnic groups may be affected. Onset of the disease usually occurs in childhood between 3 and 9 years of age. ${ }^{2-7,8}$ According to the Eurofever registry, ${ }^{9} 78 \%$ of all diagnoses of FMF correspond to individuals under 18 years of age in Western Europe. A late onset is more prevalent in males and is associated to a lesser response to colchicine therapy. ${ }^{2,3,10-12}$

FMF is an autosomal recessive (AR) hereditary disease. The Infevers database has registered and described over 380 variants of the MEFV (MEditerranean FeVer) gene, located on the short arm of chromosome 16, though not all of them are of pathogenic relevance. ${ }^{9,13,14}$ The most common variants associated with the disease are M680I, M694V, M694I, and V726A in exon 10 and E148Q in exon 2, which represent $74 \%$ of the genotypes of FMF in Arab, Turkish, Armenian, and Jewish populations., 9,15,16 The correlations between genotype and phenotype are variable and complex, though some variants are associated with increased disease severity. This is the case of M694V in homozygosis, which implies early onset of the disease with a greater tendency to develop osteoarthritis and amyloidosis, and a need for high colchicine doses to control the disorder..$^{15-17}$ The SAA (associated to amyloidosis) and MICA genes are modifiers of the disease that contribute to its severity. The personal and family history, and ethnicity have also been reported to play an important role in the origin of the disease; it is therefore believed that several of pathogenic factors remain to be identified. ${ }^{7,15}$

The diagnosis ${ }^{18-20}$ of FMF is based on three factors: the typical clinical manifestations of the disease, a positive response to colchicine therapy (Table 1), and genetic tests that confirm the diagnosis-though they do not detect all the mutations. Negative testing therefore does not necessarily discard the diagnosis. ${ }^{15,16}$

The differential diagnosis is very extensive, due to the broad range of clinical manifestations involved, and should be established mainly with other hereditary recurrent fever syndromes such as hyperimmunoglobulinemia D syndrome (HIDS), TNF receptor-associated periodic syndrome (TRAPS), and cryopyrin-associated autoinflammatory syndrome (CAPS), as well as other more frequent disorders such as irritable bowel syndrome, endometriosis, appendicitis, diverticulitis, Crohn's disease, pancreatitis, porphyria, crystal deposition osteoarthritis, lymphoma, Still's disease, PFAPA (periodic fever, aphthous stomatitis, pharyngitis, adenitis), and malaria among others. ${ }^{7,17}$

First line treatment of the disease is the administration of colchicine via oral route, ${ }^{10,21}$ indicated for the control of recurrences in the form of inflammatory crises or attacks, and also for preventing the development of secondary amyloidosis. ${ }^{6}$ Sixty percent of all patients respond to treatment, $20-30 \%$ show a partial response, and only $5-10 \%$ fail to respond to treatment. ${ }^{21-23}$ Other available drugs are the anti-interleukin-1 (anti-IL-1) agents such as anakinra administered as a daily subcutaneous dose, rilonacept administered once in a week, and canakinumab administered every 4-8 weeks. ${ }^{11,12}$

This study describes the cases of FMF monitored by the Department of Pediatrics in our center, with the aim of establishing a greater level of suspicion of the disease that can allow us to ensure early diagnosis and treatment, and thus improve the quality of life of the patients.

\section{Materials and methods}

A retrospective descriptive study was made of the cases of FMF diagnosed by Tel- Hashomer criteria in individuals under 18 years of age and monitored by the Department of Pediatrics (Hospital General de Granollers [HGG], Barcelona, Spain) between 2010 and 2018.

Our center is a district hospital serving a pediatric population of about 90,000 subjects under 18 years of age. Approximately $10 \%$ of the pediatric population corresponds to families from other countries-mostly from Morocco (55\% of the total immigrants), followed by Senegal (15\%), Romania (15\%), Bolivia (10\%), and China (7\%). ${ }^{24}$

The study included all patients under 18 years of age with diagnostic criteria of FMF (Table 1), diagnosed and monitored by the Department of Pediatrics of HGG between January 2010 and December 2018. The exclusion criteria were those cases not confirmed according to clinical criteria, patients over 18 years of age at the time of diagnosis, and/or patients without a genetic study.

The following data were compiled from the case history: age (at clinical onset of the disease, at diagnosis, and at the end of data collection), gender, ethnic origin, genetic variants, clinical manifestations, complications, laboratory tests (C-reactive protein, erythrocyte sedimentation rate $[E S R]$, amyloid $A$ ), and treatment at the start and at the end of the observation period. As this was a retrospective descriptive study, the obtainment of informed consent was not considered necessary. The genetic studies of the mutations of the MEFV gene were all made at the Immunology Laboratory of Hospital Clínic de Barcelona (Barcelona, Spain). Prior informed consent for the genetic studies was obtained from the parents and/or legal guardians in all cases.

\section{Results}

The sample consisted of seven patients (four boys and three girls) aged between 1 and 18 years at the time of the study (Table 2 ). The mean age at the time of diagnosis was 4.5 years (range: 8 months to 8 years). Detailed evaluation of the case history showed the mean patient age at the time of onset of the clinical manifestations to be 17.5 months (range: 6-30 months), representing an average diagnostic delay of 3 years. 
Table 1 Diagnostic criteria of familial Mediterranean fever.

Diagnostic criteria of Tel Hashomer:

Definitive diagnosis: Two major criteria or one major + two minor criteria

Probable diagnosis: One major criterion + One minor criterion

- Major criteria:

- Episodes of recurrent fever accompanied by serositis

- Type AA amyloidosis with no other predisposing disease

- Response to colchicine

- Minor criteria:

- Recurrent fever attacks

- Erysipela-type erythema

- History in first-degree relatives

Criteria of Livneh (simplified):

Definitive diagnosis: One major criterion or two minor criteria

- Major criteria:

- Typical generalized peritonitis attacks*a

- Unilateral pleuritis

- Pericarditis

- Single joint osteoarthritis of the elbow, knee, or ankle

- Minor criteria:

- 1-2 incomplete attacks affecting one or more sites (abdomen, lungs, leg) *b $^{*}$

- Response to colchicine

Turkish pediatric criteria

Definitive diagnosis: The presence of at least two of the following five criteria:

- Fever: axillary temperature $>38^{\circ} \mathrm{C}$, duration $6-72 \mathrm{~h} \geq 3$ attacks

- Abdominal pain: duration 6-72 $\mathrm{h} \geq 3$ attacks

- Chest pain: duration 6-72 $\mathrm{h} \geq 3$ attacks

- Osteoarthritis: duration 6-72 h, $\geq 3$ attacks and oligoarthritis

- Family history of FMF

*aTypical attacks are defined as recurrent ( $\geq 3$ of the same kind), febrile (rectal temperature $38^{\circ} \mathrm{C}\left[100^{\circ} \mathrm{F}\right]$ or higher), and brief (duration between $12 \mathrm{~h}$ and 3 days).

*b Incomplete attacks are defined as episodes differing from typical attacks in 1 or 2 characteristics, as follows: Temperature normal or $<38^{\circ} \mathrm{C}\left(100^{\circ} \mathrm{F}\right)$. The attacks are longer or shorter than indicated (but no less than $6 \mathrm{~h}$ or more than 1 week). Absence of signs of peritonitis during the abdominal attacks. Localized abdominal attacks. Osteoarthritis of joints other than those indicated.

We monitored the patients from 2010 to 2018, with a review of the case histories from the time of diagnosis to the end of the observation period. During the latter, we recorded four losses (three patients changed their healthcare center or home address, and one reached legal adult age).
Most of the patients (85\%) were Caucasians, and of these, 29\% were of Moroccan origin (Figure 1). No consanguinity was observed in our series.

All the cases initially manifested with fever-the crises lasting 3-4 days with a periodicity of 15-30 days-and this was the sole reason for consultation in $57.2 \%$ of the patients (Figure 2). Fever in turn was accompanied by other symptoms such as joint and muscle pain (57\%), skin lesions (28\%), abdominal pain (42\%), microcytic anemia (28\%), and oropharyngeal alterations (14\%). In no case were erysipela-type skin lesions reported. Of note is the fact that one of the girls presented marked sensorineural hearing loss at the time of diagnosis, and another suffered pericardial effusion at the time of onset (Figure 3).

There was great variability in the genetic variants in our patients (Table 2). All the detected variants were in heterozygosis-in one case shared with a mutation in homozygosis. The most frequently identified MEFV gene variant was E148Q in exon 2, described as variant of uncertain significance by the American College of Clinical Genetics and Genomics.

Amyloid A elevation was detected in plasma at the control tests in two of the patients (28\% of the series), at 5 and 10 years of age (Table 2). In both cases, amyloid A was seen to normalize with treatment, in one case following monotherapy with colchicine, while in the other case treatment with anakinra was required.

Initial therapy consisted of colchicine via the oral route in all cases, with variation of the dose according to the clinical course and response to treatment. The adverse effects associated to the administration of colchicine were limited to dyspeptic bowel movements at the start of therapy in three patients (42\%), which disappeared after a few weeks without intervention and/or adjusting the administered dose. At the end of the study, four of the patients remained on colchicine and showed complete control of the symptoms without adverse effects or laboratory test alterations. During follow-up, two of the patients discontinued colchicine. The first case corresponded to a girl with very early onset (at 6 months of age) in the form of pericardial effusion (Figure 3), though she subsequently presented no clinical manifestations or laboratory test alterations despite poor adherence to therapy. Monitoring without treatment was therefore decided, and the patient remained symptoms-free until the end of the study, at 12 years of age. The second case corresponded to a boy at 5 years of age manifested with recurrent fever and amyloid A elevation. The condition improved with colchicine, though the latter was subsequently suspended due to poor gastrointestinal tolerance, with no clinical relapse. Both cases corresponded to variants of uncertain significance in a single allele in heterozygosis (E148Q and I591T, respectively).

Lastly, one girl responded favorably to colchicine, which she started at 8 years of age, though during the follow-up she suffered important clinical worsening with elevation of all the acute phase reactants and amyloid A that required the start of anakinra at 14 years of age, with a good response. However, a few months later, she suffered anaphylactic shock and was admitted to intensive care. The treatment was therefore suspended, and 


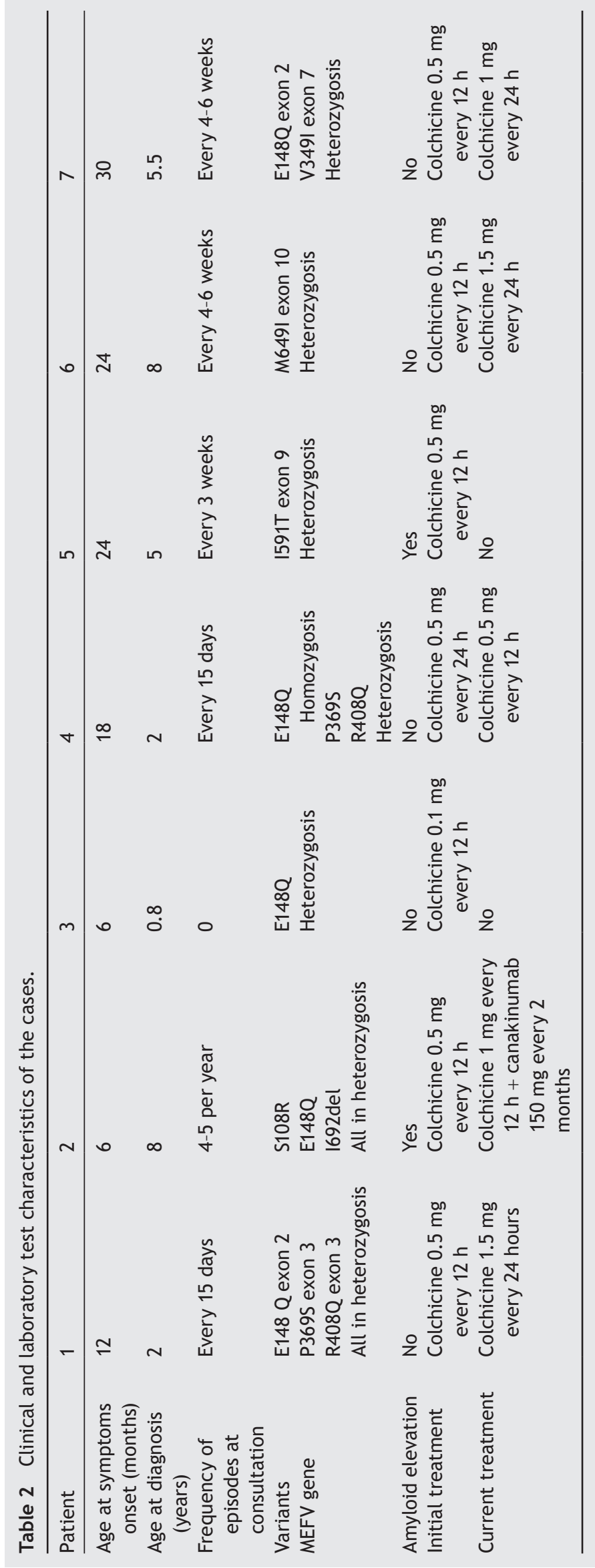




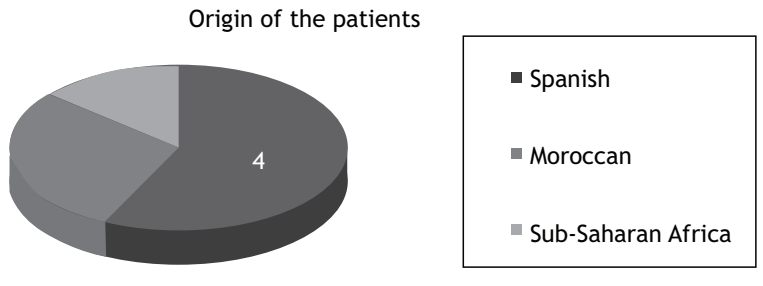

Figure 1 Origin of the patients.

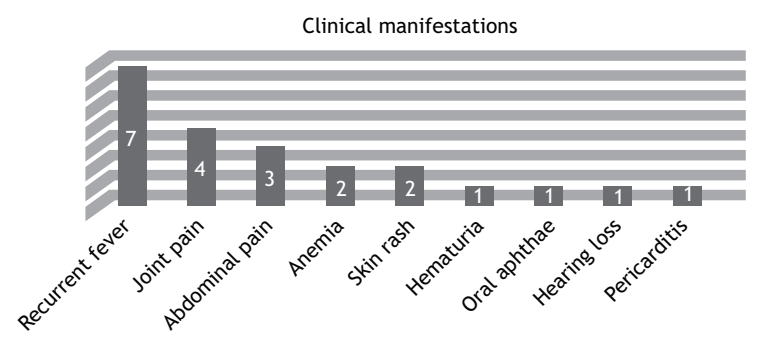

Figure 2 Clinical manifestations at diagnosis.

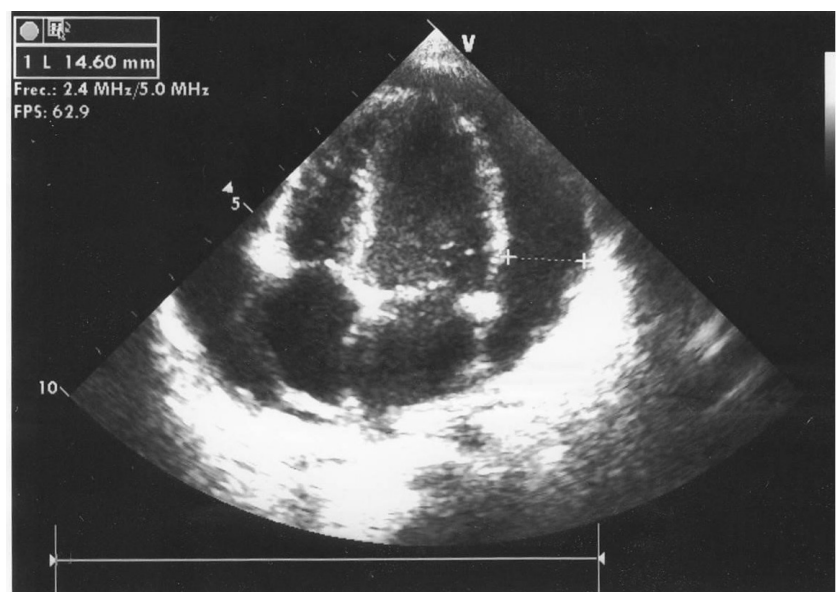

Figure 3 Pericardial effusion as initial manifestation in a female infant with variant in heterozygosis; currently asymptomatic.

canakinumab was required for control of the disease. This patient is currently 18 years old and remains on treatment with canakinumab every 8 weeks plus daily colchicine, with good tolerance, no symptoms, and laboratory test parameters within normal limits.

\section{Discussion}

In the present series, the first clinical manifestation was recurrent fever in all patients. Indeed, in most cases (57.2\%), fever was the sole reason for medical consultation, and in the rest of the cases, fever was accompanied by other manifestations (42.8\%): joint pain in four patients, abdominal pain in two, and pericardial effusion in one case. It should be noted that recurrent fever was the only manifestation in a considerable number of patients, which allowed us to establish an early diagnosis and improve the quality of life of these children.

An interesting observation on examining the family history of our cases was the absence of consanguinitythough it must be noted that all our patients were of Mediterranean origin. Regarding ethnicity and considering the limitations imposed by the small sample size, two of the children (28\%) were of Moroccan origin, and only four were of European origin (57\%). This is notorious, since individuals of Moroccan origin represent only $5 \%$ of the global population in our setting, while those of European origin account for a full $90 \%$. We thus underscore the need to suspect these disorders even in the absence of consanguinity and in all ethnic groups-without forgetting the high prevalence of FMF in the mentioned population groups.

On the other hand, in two of the patients that initially responded well to colchicine, the latter could be suspended (due to poor adherence in one case and poor tolerance in the other) without reappearance of the clinical manifestations. Both patients presented a single variant classified as being of uncertain significance, though they met the criteria for establishing a clinical diagnosis. In one of them, first treatment with colchicine normalized initial amyloid A protein plasmatic elevation, with no subsequent reappearance of clinical or laboratory test anomalies up until the end of the study. This suggests the possibility of greater clinical manifestations among smaller school and preschool children versus older patients. On the other hand, and although further confirmatory data are needed, this observation suggests the possibility of patient follow-up without treatment for a period in children with a single variant of uncertain significance and a good clinical course-though with close clinical and laboratory test monitoring to detect any possible worsening.

On analyzing the amyloid A plasma values, we recorded elevation of the protein in two patients at a very early age (5 and 10 years). The oral colchicine dose was thus incremented, with improvement in both cases. The younger patient showed normalization of the values that persisted even after discontinuation of the treatment. In the other case, normalization was not achieved, and anakinra had to be added to therapy to control the associated clinical manifestations and normalize the amyloid $A$ values. The rest of the patients did not show amyloid $A$ elevation and evolved favorably with colchicine throughout the study period.

Treatment with anti-IL-1 medication was only prescribed in one patient (a girl), with a good response to both anakinra and canakinumab. We initially introduced treatment in the form of anakinra, with good control of the clinical manifestations and laboratory test values in 6 months. Suspension was subsequently required due to the appearance of a serious adverse reaction, with reappearance of the clinical manifestations 1 month after discontinuing anakinra. We therefore started canakinumab, with a good response and tolerance that persisted for over 2 years at the time of closure of the study.

The follow-up period of our series during these 9 years covers a cumulative total of 462 months, and in no case was renal failure detected. The oldest patient at the end of the study was 18 years old, and the age of our series therefore might explain the absence of renal failure. Nevertheless, 
we wish to underscore the importance of early diagnosis and treatment, because this is crucial to control the inflammatory parameters associated with a poor prognosis and to improve the clinical situation of the patient.

\section{Conclusions}

In our series, the onset of clinical manifestations of FMF occurred before 30 months of age in all cases-the most common symptoms being recurrent fever, joint pain, and abdominal pain. Clinical suspicion and genetic study allow an early diagnosis, which are crucial to provide effective treatment, which improves the prognosis and quality of life of the patients.

It is very important to consider the possibility of complications such as secondary amyloidosis and the need to assess clinical and laboratory periodic controls including amyloid A plasma levels and renal function even in pediatric cases.

Treatment with colchicine is effective in most patients, though treatment with anti-IL-1 drugs should be considered and prescribed also in pediatric patients.

\section{Conflicts of interest}

The authors declare that they have no potential conflicts of interest with respect to research, authorship, and/or publication of this article.

\section{References}

1. Shohat M. Familial Mediterranean fever. In: Adam MP, Ardinger HH, Pagon RA, et al., editors. GeneReviews $₫$ [Internet]. 2000 (Updated 2016 Dec 15). Available from: https://www.ncbi. nlm.nih.gov/books/NBK1227/ [Accessed 21 February 2021].

2. Marek-Yagel D, Berkun $Y$, Padeh S. Clinical disease among patients heterozygous for familial Mediterranean fever. Arthritis Rheum. 2009;60:1862. http://dx.doi.org/10.1002/ art.24570

3. Ben-Chetrit E, Touitou I. Familial Mediterranean fever in the world. Arthritis Rheum. 2009;61:1447. http://dx.doi.org/10. 1002/art.24458

4. Infevers: An online database for autoinflammatory mutations [Internet]. Available from: https://infevers.umai-montpellier. $\mathrm{fr} /$ [Accesed May 2021].

5. Arostegui Jl. Hereditary systemic autoinflammatory diseases. Reumatol Clin. 2011;7(1):45-50. http://dx.doi.org/10.1016/j. reuma.2010.01.010

6. Westermark GT, Fändrich $M$, Westermark P. AA amyloidosis: Pathogenesis and targeted therapy. Annu Rev Pathol. 2015;10:321. http://dx.doi.org/10.1146/annurev-pathol-020712163913

7. Gattorno M, Hofer M, Federici S. Classification criteria for autoinflammatory recurrent fevers. Ann Rheum Dis. 2019;78(8):1025-32. http://dx.doi.org/10.1136/annrheumdis2019-215048

8. Özen S. Update on the epidemiology and disease outcome of familial Mediterranean fever. Best Pract Res Clin Rheumatol. 2018;32:254-60. http://dx.doi.org/10.1016/j.berh.2018.09.003
9. Eurofever registry [Internet]. Available from: https://www. printo.it/eurofever/score_criteria [Accessed 21 April 2021].

10. Ozen S, Demirkaya E, Erer B, Livneh A, Ben-Chetrit E, Giancane G. EULAR recommendations for the managementof familial Mediterranean fever. Ann Rheum Dis. 2016;75:644-51. http://dx.doi.org/10.1136/annrheumdis-2015-208690

11. De Benedetti F, Gattorno M, Anton J, Ben-Chetrit E, Frenkel J, Hoffman HM. Canakinumab for the treatment of autoinflammatory recurrent fever syndromes. N Engl J Med. 2018;378:1908-19. http://dx.doi.org/10.1056/ NEJMoa1706314

12. Bayram MT, Çankaya T, Bora E. Risk factors for subclinical inflammation in children with Familial Mediterranean fever. Rheumatol Int. 2015;35:1393. http://dx.doi.org/10.1007/ s00296-015-3227-z

13. Arpacı A, Doğan S, Erdoğan HF, El Ç, Cura SE. Presentation of a new mutation in FMF and evaluating the frequency of distribution of the MEFV gene mutation in our region with clinical findings. Mol Biol Rep. 2021 Mar;48(3):2025-33. http://dx.doi. org/10.1007/s11033-020-06040-y

14. Dode C, Pecheux C, Cazeneuve C, Cattan D, Dervichian M, Goossens M. Mutations in the MEFV gene in a large series of patients with a clinical diagnosis of familial Mediterranean fever. Am J Med Genet. 2000;92:241-6. http://dx.doi. org/10.1002/(SICI)1096-8628(20000605)92:4\%3C241::AIDAJMG3\%3E3.0.CO;2-G

15. Shinar Y, Livneh A, Langevitz P. Genotype-phenotype assessment of common genotypes among patients with familial Mediterranean fever. J Rheumatol. 2000;27:1703.

16. Giancane G, Ter Haar NM, Wulffraat N, Vastert SJ, Barron K, Hentgen V. Evidence-based recommendations for genetic diagnosis of familial Mediterranean fever. Ann Rheum Dis. 2015 Apr;74(4):635-41. http://dx.doi.org/10.1136/annrheumdis-2014206844

17. Touitou I. The spectrum of familial Mediterranean Fever (FMF) mutations. Eur J Hum Genet. 2001;9(7):473-83. http://dx.doi. org/10.1038/sj.ejhg.5200658

18. Yalçinkaya F, Ozen S, Ozçakar ZB, Aktay N, Cakar N, Düzova A. A new set of criteria for the diagnosis of familial Mediterranean fever in childhood. Rheumatology (Oxford). 2009 Apr;48(4):395-8. http://dx.doi.org/10.1093/ rheumatology/ken509

19. Ozçakar ZB, Yalçınkaya F, Cakar N. Application of the new pediatric criteria and Tel Hashomer criteria in heterozygous patients with clinical features of FMF. Eur J Pediatr. 2011;170:1055. http://dx.doi.org/10.1007/s00431-011-1404-y

20. Berkun Y, Eisenstein EM. Diagnostic criteria of familial Mediterranean fever. Autoimmun Rev. 2014 Apr-May;13(45):388-90. http://dx.doi.org/10.1016/j.autrev.2014.01.045

21. Polat A, Acikel C, Sozeri B, Dursun I, Kasapcopur O, Gulez N. Comparison of the efficacy of once- and twice-daily colchicine dosage in pediatric patients with familial Mediterranean fever-A randomized controlled noninferiority trial. Arthritis Res Ther. 2016;18:85. http://dx.doi.org/10.1186/s13075-016-0980-7

22. Özen S, Kone-Paut I, Gül A. Colchicine resistance and intolerance in familial Mediterranean fever: Definition, causes, and alternative treatments. Semin Arthritis Rheum. 2017;47:115-20. http://dx.doi.org/10.1016/j.semarthrit.2017. 03.006

23. Eroglu FK, Besbas N, Topaloglu R, Özen S. Treatment of colchicine-resistant familial Mediterranean fever in children and adolescents. Rheumatol Int. 2015;35:1733-7. http://dx.doi. org/10.1007/s00296-015-3293-2

24. Idescat. [Internet]. Available from: https://www.idescat. es/poblacioestrangera/?b=1\&geo=cat\&nac=a\#Plegable=geo [Accesed May 2021]. 